\title{
Erratum
}

\section{Erratum to "Therapeutic Effectiveness and Safety of Mesotherapy in Patients with Osteoarthritis of the Knee"}

\author{
Liang Chen, ${ }^{1}$ Dongqing Li, ${ }^{2}$ Jun Zhong, ${ }^{1}$ Bo Qiu $\mathbb{D},{ }^{1}$ and Xianglei Wu $\mathbb{D}^{3,4}$ \\ ${ }^{1}$ Department of Orthopaedics, Renmin Hospital of Wuhan University, 9 Zhangzhidong Street, Wuhan, Hubei 430060, China \\ ${ }^{2}$ Department of Microbiology, School of Basic Medical Science, Wuhan University, 185 Donghu Road, Wuhan, Hubei 430071, China \\ ${ }^{3}$ Institute of ENT, EENT Hospital of Fudan University, 83 Fenyang Road, Shanghai 300021, China \\ ${ }^{4}$ Laboratory of Immunology, University of Lorraine, avenue du Morvan, 54511 Vandoeuvre-lès-Nancy, France
}

Correspondence should be addressed to Bo Qiu; qbtg163@163.com and Xianglei Wu; wxlthibaut@gmail.com

Received 10 April 2018; Accepted 22 April 2018; Published 14 May 2018

Copyright (C) 2018 Liang Chen et al. This is an open access article distributed under the Creative Commons Attribution License, which permits unrestricted use, distribution, and reproduction in any medium, provided the original work is properly cited.

In the article titled "Therapeutic Effectiveness and Safety of Mesotherapy in Patients with Osteoarthritis of the Knee" [1], Dr. Bo Qiu should be listed as a corresponding author along with Xianglei Wu.

\section{References}

[1] L. Chen, D. Li, J. Zhong, B. Qiu, and X. Wu, "Therapeutic effectiveness and safety of mesotherapy in patients with osteoarthritis of the knee," Evidence-Based Complementary and Alternative Medicine, vol. 2018, Article ID 6513049, 6 pages, 2018. 


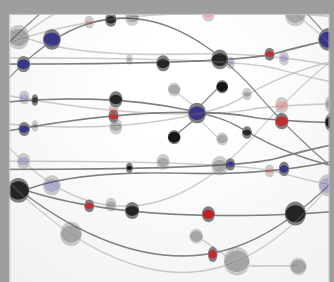

The Scientific World Journal
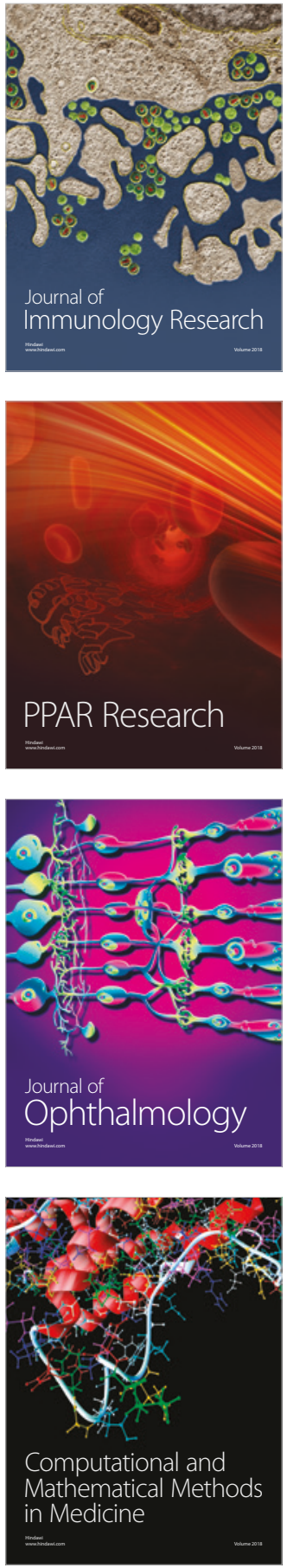

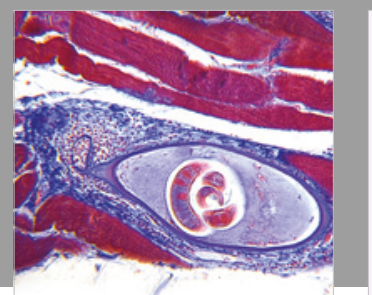

Gastroenterology Research and Practice

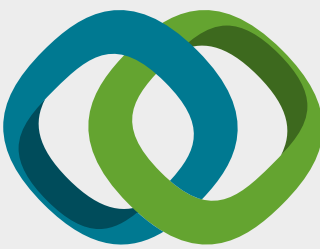

\section{Hindawi}

Submit your manuscripts at

www.hindawi.com
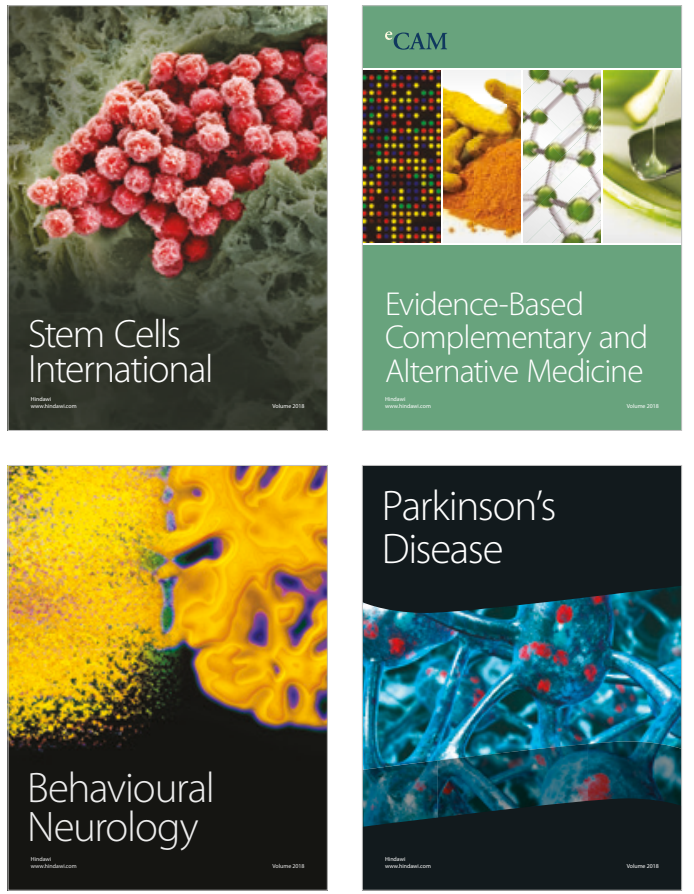

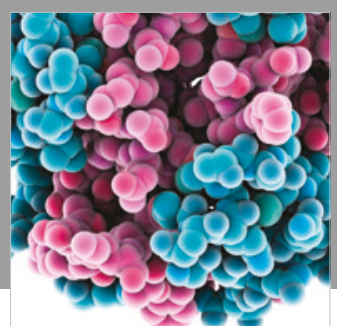

ournal of

Diabetes Research

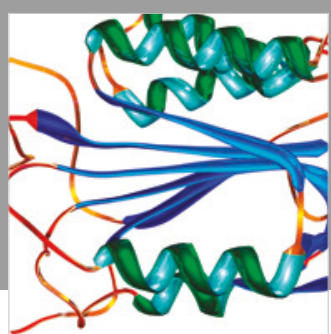

Disease Markers
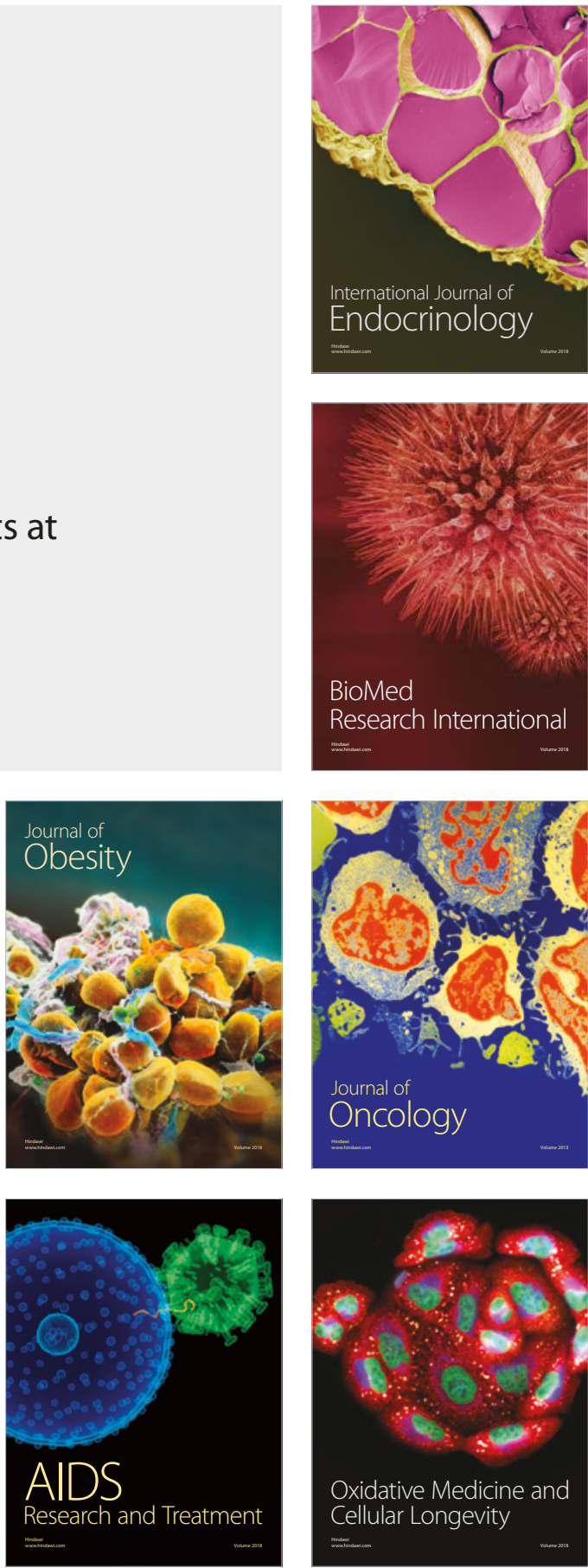